\title{
Comparação sobre a eficácia e sensibilidade dos diferentes tipos de clareamento dental: uma revisão de literatura
}

\author{
Comparison on the effectiveness and sensitivity of different types of tooth whitening: a literature \\ review
}

Comparación de la efectividad y sensibilidad de diferentes tipos de blanqueamiento dental: revisión de la literatura

\section{Resumo}

Nos dias atuais, a estética do sorriso vem crescendo no mercado, visto que muitos consideram como parte do padrão de beleza desejável, o sorriso alinhado e os dentes brancos. Assim sendo, o clareamento dental constitui um dos procedimentos mais procurados e realizados pelos cirurgiões-dentistas, no quesito estética. Dessa forma, o cirurgiãodentista deve ter uma base sólida de conhecimento a respeito, para que faça uma avaliação clínica e o correto diagnóstico da estrutura dental, indicando a melhor técnica em cada caso. Para tanto, foi realizada uma revisão de literatura sobre os tipos de clareamento dental, comparando-os quanto à eficácia, em relação à alteração de cor, e quanto à sensibilidade, com buscas nas bases de dados, Pubmed, Scielo e Scopus, através dos descritores: Tooth bleaching; Carbamide peroxide; Hydrogen Peroxide; Dentin Sensitivity. Foram considerados elegíveis estudos em inglês, português ou espanhol, publicados entre 01/2006 a 05/2021. Assim, notou-se que as técnicas de clareamento são capazes de atingir os mesmos resultados quanto à mudança de cor, mas com variação no tempo e indicação. Quanto à sensibilidade, há uma tendência dessa ser maior, quando usado gel de maior concentração. Porém, já existem 
atualmente, géis clareadores contendo substâncias dessensibilizantes, e/ou protocolos que podem ser utilizados, para que se tenha uma redução da mesma. Além disso, quando realizada a técnica de clareamento caseiro, sugere-se dar preferência ao gel de Peróxido de Carbamida na menor concentração, uma vez que esse apresenta menor risco de desenvolvimento de sensibilidade dentária e é eficaz, se comparado às maiores concentrações.

Palavras-chave: Clareamento dental; Peróxido de carbamida; Peróxido de hidrogênio; Sensibilidade da dentina.

\begin{abstract}
Nowadays, the aesthetics of the smile has been growing in the market, since many consider the aligned smile and white teeth as part of the desirable beauty standard. Therefore, tooth whitening is one of the most sought after procedures performed by dentists, in terms of aesthetics. Thus, the dentist must have a solid base of knowledge about it, so that they can make a clinical assessment and correct diagnosis of the dental structure, indicating the best technique in each case. To this end, a literature review was carried out on the types of tooth whitening, comparing them in terms of efficacy, in relation to color change, and in terms of sensitivity, with searches in the databases, Pubmed, Scielo and Scopus, through the Keywords: Tooth bleaching; Carbamide peroxide; Hydrogen Peroxide; Dentin Sensitivity. Studies in English, Portuguese or Spanish, published between 01/2006 and 05/2021, were considered eligible. Thus, it was noted that bleaching techniques are able to achieve the same results regarding color change, but with variation in time and indication. As for sensitivity, there is a tendency for this to be greater when using a higher concentration gel. However, there are already bleaching gels containing desensitizing substances, and/or protocols that can be used, so that it can be reduced. In addition, when the at-home whitening technique is performed, it is suggested to give preference to Carbamide Peroxide gel in the lowest concentration, since this presents a lower risk of developing tooth sensitivity and is effective, when compared to higher concentrations.
\end{abstract}

Keywords: Tooth bleaching; Carbamide peroxide; Hydrogen peroxide; Dentin sensitivity.

\title{
Resumen
}

Hoy en día, la estética de la sonrisa ha ido creciendo en el mercado, ya que muchos consideran la sonrisa alineada y los dientes blancos como parte del estándar de belleza deseable. Por tanto, el blanqueamiento dental es uno de los procedimientos más solicitados que realizan los dentistas, en cuanto a estética. Así, el odontólogo debe tener una base sólida de conocimientos al respecto, para que pueda realizar una valoración clínica y un correcto diagnóstico de la estructura dentaria, indicando la mejor técnica en cada caso. Por ello, se realizó una revisión de la literatura sobre los tipos de blanqueamiento dental, comparándolos en cuanto a eficacia, en relación al cambio de color, y en cuanto a sensibilidad, con búsquedas en las bases de datos Pubmed, Scielo y Scopus, a través de las Palabras clave: Tooth bleaching; Carbamide peroxide; Hydrogen Peroxide; Dentin Sensitivity. Se consideraron elegibles los estudios en inglés, portugués o español, publicados entre el 01/2006 y el 05/2021. Así, se observó que las técnicas de blanqueo pueden lograr los mismos resultados en cuanto al cambio de color, pero con variación en el tiempo y la indicación. En cuanto a la sensibilidad, existe una tendencia a que sea mayor cuando se utiliza un gel de mayor concentración. Sin embargo, ya existen geles blanqueadores que contienen sustancias desensibilizantes, y / o protocolos que se pueden utilizar para que se pueda reducir. Además, cuando se realiza la técnica de blanqueamiento domiciliario, se sugiere dar preferencia al gel de Peróxido de Carbamida en la concentración más baja, ya que éste presenta un menor riesgo de desarrollar sensibilidad dental y es efectivo, en comparación con concentraciones más altas.

Palabras clave: Blanqueamiento de dientes; Peróxido de carbamida; Peróxido de hidrógeno; Sensibilidad de la dentina.

\section{Introdução}

O clareamento existe desde o Egito antigo, onde utilizavam-se abrasivos e vinagre a fim de clarear os dentes, e também onde os romanos utilizavam urina para o mesmo fim. Heywood e Heymann, em 1989, introduziram a técnica de clareamento dental com moldeiras, tendo como agente clareador o Peróxido de Carbamida a 10\%, no período noturno por quinze dias. Essa técnica popularizou-se por conta de sua praticidade e eficácia, e posteriormente foi adaptada a maiores concentrações e ao uso da moldeira durante o dia (Niessen, 2007).

Para que haja sucesso no tratamento clareador, o cirurgião-dentista deve conhecer os diferentes tipos de manchamentos e saber diagnosticar a etiologia das alterações de cor, pois o prognóstico poderá ser favorável ou desfavorável, dependendo da causa do manchamento (Araujo, Reis, Gonçalves, \& Brum, 2015; Mchantaf, Mansour, Sabbagh, Feghali, \& Mcconnell, 2017; Moradas, 2017; Niessen, 2007).

Existem basicamente, dois tipos de alterações de cor, as causadas por fatores extrínsecos e as causadas por fatores intrínsecos (congênitos ou adquiridos). Assim sendo, como manchamentos extrínsecos pode-se citar aqueles causados por chá, 
café, cigarro, bebidas ou alimentos com corantes artificiais, e o acúmulo de placa. Já como manchas intrínsecas congênitas pode-se citar, dentinogênese imperfeita e fluorose; e as adquiridas, que subdividem-se em pré e pós-eruptivas sendo, por exemplo, tetraciclinas e flúor; traumatismos e tetraciclinas, respectivamente. Há também causas iatrogênicas, como por exemplo, o emprego inadequado de alguns materiais para a obturação dos condutos radiculares e/ou para restaurar cavidades de acesso endodôntico; ou ainda a abertura inadequada da coroa durante o acesso, permitindo a presença de restos necróticos de polpa ou propiciando o acúmulo de material selador nesta região (Baratieri et al., 1993). Além disso, a descoloração devido ao envelhecimento pode ser considerada intrínseca, uma vez que à medida que os dentes envelhecem, forma-se mais dentina secundária e a camada de esmalte torna-se mais estreita e translúcida (Mchantaf et al., 2017).

O mecanismo de ação dos agentes clareadores consiste na oxidação de compostos orgânicos e na liberação de radicais livres capazes de promover a quebra dos pigmentos, transformando-os em compostos mais claros. À medida que o clareamento prossegue, chega-se a um ponto em que há apenas estruturas incolores hidrofílicas, esse é o chamado ponto de saturação. O clareamento diminui drasticamente e o processo começa a quebrar a estrutura de carbono das proteínas, e outros materiais que contenham carbono. Portanto, é fundamental que o cirurgião-dentista saiba que o clareamento deve ser interrompido antes ou no ponto de saturação (Araujo et al., 2015; Mchantaf et al., 2017). O Peróxido de Carbamida é muito instável e, portanto, se decompõe em Peróxido de Hidrogênio e uréia. O Peróxido de Hidrogênio se decompõe ainda em oxigênio e água, ao passo que a uréia se decompõe em amônia e dióxido de carbono. O Peróxido de Hidrogênio é considerado o agente ativo, enquanto a uréia tem um papel importante na elevação do pH (Baratieri et al., 1993). Assim sendo, conhecer a concentração de Peróxido de Carbamida permite calcular a concentração do Peróxido de Hidrogênio ativo na superfície do dente, pois sabe-se que a relação entre eles é de 1/3. Quando o Peróxido de Hidrogênio se decompõe em água e oxigênio, o oxigênio é que penetra no dente e libera a molécula de pigmento, levando ao clareamento (Mchantaf et al., 2017).

O clareamento dental pode ser realizado no consultório, pelo cirurgião-dentista, utilizando concentrações mais altas de Peróxido de Hidrogênio; em casa, utilizando moldeiras individuais com Peróxido de Carbamida ou Hidrogênio, em concentrações mais baixas (Almeida et al., 2021; Rodrigues et al., 2018); ou ainda a combinação do tratamento em casa e no consultório (Mchantaf et al., 2017). Porém, os clareadores, especialmente aqueles em altas concentrações, podem causar uma sensibilidade temporária aos dentes e gengivas. Assim sendo, um agente dessensibilizante fornecido pelo dentista pode ajudar, e a interrupção do uso do agente por um ou dois dias também pode minimizar a sensibilidade (Niessen, 2007). Inclusive já existem protocolos dessensibilizantes e géis clareadores que possuem em sua formulação algum agente remineralizante, como por exemplo, nitrato de potássio e fluoreto de sódio, ou cálcio e fluoreto, respectivamente (Almeida et al., 2021; Gonçalves et al., 2017; Meireles, Santos, Bona \& Demarco, 2009; Rahal et al., 2015; Silva, Nacano \& Gava Pizi, 2012; Vieira et al., 2020).

Vale ressaltar que o clareamento não altera a cor das restaurações presentes nos dentes, portanto, elas podem parecer mais escuras após o procedimento. Além disso, os cirurgiões-dentistas geralmente recomendam o clareamento antes da colocação de facetas para mudar a cor ou a posição dos dentes anteriores (Niessen, 2007). Porém, como o clareamento é capaz de reduzir significativamente a força de união das resinas compostas com o esmalte condicionado, caso seja necessário fazer alguma restauração adesiva após o procedimento, recomenda-se aguardar cerca de 7 a 14 dias do término ou interrupção do clareamento (Baratieri et al., 1993; Mchantaf et al., 2017).

Dessa forma, nota-se que os principais fatores que afetam o sucesso do clareamento são: a cor inicial dos dentes, a capacidade de resposta do paciente, a concentração do material (Mchantaf et al., 2017), e o tempo de aplicação (Niessen, 2007). Como visto, atualmente existem três protocolos de clareamento dental: de consultório, caseiro, ou a associação das técnicas. Assim sendo, o cirurgião-dentista deve ter em vista as indicações, vantagens e desvantagens de cada um deles, para escolher qual o melhor protocolo a ser utilizado em cada situação clínica. Portanto, diante dos protocolos disponíveis, torna-se oportuno uma revisão da literatura a fim de avaliar a eficácia dos mesmos, em relação à alteração de cor e também quanto à 
possível sensibilidade dentária, para que possamos auxiliar os profissionais na busca de melhores resultados e satisfação dos pacientes.

\section{Metodologia}

O estudo constitui uma revisão narrativa da literatura, com pesquisa de natureza qualitativa e suporte metodológico de Estrela (2018), comparando os diferentes tipos de clareamento dental quanto à eficácia, em relação à alteração de cor, e sensibilidade dentária. A busca dos artigos foi realizada nas bases de dados Pubmed, Scielo e Scopus, com as palavras-chave, "Tooth bleaching", "Carbamide peroxide", "Hydrogen Peroxide", "Dentin Sensitivity” e seus respectivos correspondentes em português. Foram considerados elegíveis estudos em inglês, português ou espanhol, publicados entre 01/2006 a 05/2021. Foram incluídos artigos de revisão sistemática e meta-análise, revisão de literatura, e pesquisas in vitro e in vivo, que comparassem as técnicas de clareamento, ou trouxessem dados sobre as técnicas separadamente. Foram excluídos artigos em que o tema fugisse à proposta da revisão, artigos no qual o resumo apresentava acesso restrito, resumo com acesso disponível e artigo restrito, além de comentários, editoriais, cartas, diretrizes das sociedades médicas e artigos que não apresentaram relação com o tema do manuscrito. No total, foram incluídos 30 artigos. As referências bibliográficas dos artigos incluídos também foram consultadas, em busca de outros estudos que possam contemplar os objetivos dessa revisão de literatura.

\section{Resultados e Discussão}

\subsection{Indicações e contraindicações do clareamento dental}

Quando se pensa em clareamento dental caseiro e de consultório, deve-se ter em mente, além das suas indicações, quais as vantagens e desvantagens de cada uma das técnicas. A técnica de consultório não depende da colaboração do paciente e o resultado é imediato (Araujo et al., 2015). Porém, sua desvantagem é que este é mais caro. O caseiro demonstra os primeiros resultados após cerca de cinco a sete dias, e é recomendado para o uso por duas semanas (Niessen, 2007).

Ao avaliar as indicações e contraindicações de cada tipo de clareamento, deve-se pensar a respeito da influência dos peróxidos utilizados sobre a superfície cerâmica visto que, comumente, os pacientes apresentam restaurações, coroas ou facetas cerâmicas. Assim sendo, durante uma pesquisa sobre o efeito do Peróxido de Carbamida 16\% nas propriedades da superfície da cerâmica vitrificada, observou-se que agentes clareadores de alta concentração de Peróxido de Carbamida para clareamento caseiro afetam significativamente sua rugosidade. Portanto, as restaurações cerâmicas devem ser protegidas antes de qualquer clareamento e desta forma, pacientes com restaurações cerâmicas completas, como E-max CAD e Empress CAD, devem ter cuidado ao realizar o clareamento caseiro. Além disso, pode haver necessidade de um polimento em restaurações que acidentalmente forem expostas aos géis. Ademais, a pequena mudança de dureza significativa pode levar a outras alterações, como a descoloração dos materiais (Demir, Karci \& Ozcan, 2020).

Uma dúvida muito comum entre os pacientes, quando pensam em realizar o clareamento dos dentes, é se necessitarão de aderir a uma dieta branca, para não influenciar na eficácia do tratamento. De acordo com uma pesquisa sobre a suscetibilidade do esmalte à coloração de café e vinho tinto em diferentes intervalos decorridos do clareamento com Peróxido de Hidrogênio a $35 \%$, observou-se que o café não consegue manchar a superfície do esmalte nem aos 30, nem aos 150min após a terapia. Porém, o vinho tinto é capaz de manchar o esmalte clareado aos 30 e aos 150min após o procedimento (Liporini et al., 2010). Outro estudo sobre a exposição ao café durante o clareamento caseiro com Peróxido de Carbamida a 16\% mostrou que o mesmo não afeta a sua eficácia, nem a sensibilidade dentária (Rezende, Loguercio, Reis, \& Kossatz, 2013). Entretanto, outra pesquisa mostrou que a adesão à dieta branca durante o processo de clareamento dental não melhora o resultado estético e, desta forma, não é necessária. No entanto, deve-se tomar cuidado após o clareamento, pois ocorrerá coloração extrínseca do 
esmalte clareado pelo consumo de agentes como café, chá, vinho e frutas escuras (Matias, Wang, Matis, Cook \& Eckert, 2015).

A recidiva da cor pode ocorrer por alimentos e refrigerantes que contêm pigmentos escuros, pois são absorvidos pelos dentes. Porém, mesmo que o paciente evite corantes, poderá haver recidiva. Além disso, os hábitos de higiene precários e tabagismo têm efeitos mais negativos na longevidade do clareamento (Mchantaf et al., 2017; Monteiro, Vieira, Magalhães, Silva \& Albuquerque, 2020; Niessen, 2007). Os dentes voltarão à sua cor pré-clareada em três a quatro anos, mas para impedir que essa recidiva ocorra, pode-se fazer o retratamento periódico dos dentes. Normalmente, esse é realizado com uma sessão em consultório, ou uma sequência de três semanas de uso da moldeira em casa, uma vez ao ano (Mchantaf et al., 2017).

\subsection{Clareamento dental e sensibilidade}

Durante o processo de clareamento, o esmalte sofre uma redução na microdureza (Fernandes, Strazzi-Sahyon, Suzuki, Briso, \& Santos, 2020; Mchantaf et al., 2017; Ribeiro et al., 2019), que pode ser atribuída à dissolução dos componentes de fosfato de cálcio do esmalte. Mas, uma vez terminado o processo de clareamento, ocorre uma recalcificação, preenchendo assim os espaços criados pela dissolução dos prismas de esmalte. Porém, ainda não se sabe se o esmalte recalcificado possui a mesma qualidade da matriz do esmalte, originalmente depositada (Mchantaf et al., 2017). Mas, existem géis clareadores comerciais contendo agentes remineralizantes, como cálcio ou fluoreto, que promovem a mesma eficácia de clareamento como os géis convencionais, entretanto, minimizam os efeitos negativos causados em relação à microdureza do esmalte (Palma et al., 2021; Vieira et al., 2020).

Os agentes clareadores, especialmente aqueles em altas concentrações, podem causar uma sensibilidade temporária aos dentes e gengivas (Almeida et al., 2021; Niessen, 2007), sendo que esta varia de leve a insuportável e pode diferir de um paciente para outro (Mchantaf et al., 2017). Porém, um agente dessensibilizante/remineralizante fornecido ou aplicado pelo cirurgião-dentista, pode ajudar na sensibilidade, como por exemplo, o Dessensibilize KF 2\% (FGM), que possui em sua composição 2\% de fluoreto de sódio e 5\% de nitrato de potássio (Almeida et al., 2021; Niessen, 2007; Palma et al., 2021; Rahal et al., 2015). Além disso, os dessensibilizantes podem prevenir possíveis efeitos deletérios no esmalte causados pelo clareamento prolongado (Ribeiro et al., 2019). A interrupção do uso do por um ou dois dias também pode minimizar essa sensibilidade (Niessen, 2007) e a adição de cálcio nos géis clareadores pode também auxiliar na redução da mesma (Gonçalves et al., 2017). Há ainda o clareamento de consultório com iBrite (Peróxido de Hidrogênio a 24\%). Esse possui um sistema de barril duplo que mistura os produtos ativos imediatamente antes de cada tratamento, reduzindo assim a sensibilidade sem comprometer a eficácia (Lan et al., 2019).

Em uma revisão sistemática com meta-análise sobre o risco e intensidade de sensibilidade dentária após o clareamento com um gel contendendo dessensibilizante e outro sem, observou-se que a incorporação de dessensibilizantes no gel clareador não reduziu o risco de sensibilidade, e a qualidade dessas evidências foi considerada moderada. Por outro lado, a intensidade da sensibilidade dentária, mudança de cor e risco de irritação gengival foram semelhantes entre os grupos, mas a qualidade das evidências para esses resultados foi classificada como baixa ou muito baixa, reduzindo assim o nível de confiança nesses resultados. Isso indica que são necessários mais ensaios clínicos randomizados com grandes tamanhos de amostra e uma metodologia rigorosa para confirmar esses resultados (Rezende et al., 2019).

\subsection{Clareamento de consultório $X$ clareamento caseiro}

Quanto ao clareamento de consultório, normalmente são utilizados os géis à base de Peróxido de Hidrogênio ou de Carbamida em altas concentrações, porém em um tempo reduzido de aplicação. Assim sendo, há uma discussão sobre qual a melhor forma de se aplicar esse gel no consultório, sendo uma aplicação única durante o tempo recomendado pelo fabricante, 
ou múltiplas aplicações de tempos reduzidos, na mesma sessão. Em uma pesquisa realizada sobre essa questão, verificou-se que a aplicação de um gel de Peróxido de Hidrogênio a 38\% por 40min, sem ser renovado, mantem a eficácia, o mesmo grau de clareamento e sensibilidade dentária, em comparação com duas aplicações de 20min recomendadas pelo fabricante. Logo, deve-se utilizar de preferência uma aplicação de 40min, pois a mesma não requer a renovação do gel (Martins et al., 2018). Outra pesquisa comparando a eficácia do Peróxido de Carbamida a 37\%, com o Peróxido de Hidrogênio a 35\%, notou que o clareamento com Peróxido de Carbamida a 37\% em uma única aplicação de 40min resultou em riscos reduzidos e nível de sensibilidade dental com valores próximos de zero, apesar da mudança de cor reduzida quando comparada com a obtida com Peróxido de Hidrogênio a 35\% (Peixoto et al., 2018).

Por outro lado, uma pesquisa sobre os efeitos do $\mathrm{pH}$ e da técnica de aplicação dos géis de Peróxido de Hidrogênio, no clareamento de consultório, com o objetivo de verificar a penetração do gel na câmara pulpar, mostrou que a quantidade de Peróxido de Hidrogênio que atinge a câmara pulpar foi menor quando foram usados géis de $\mathrm{pH}$ neutro/alcalino, independente da técnica de aplicação. Para géis de pH ácido, é preferível utilizar a técnica de aplicação 3 x 15min, principalmente porque a técnica 1 x 45min resulta em pH mais baixo (Balladares et al., 2019).

O clareamento caseiro, por sua vez, pode ser realizado com géis de Peróxido de Carbamida ou de Hidrogênio em concentrações mais baixas, porém, por um maior tempo de uso. Quando se compara o clareamento caseiro com Peróxido de Hidrogênio a 3,5\% e Peróxido de Carbamida a 10\%, nota-se que ambos possuem comportamento clínico uniforme, atingindo um grau satisfatório de clareamento e sem sensibilidade dentária notável (Caballero, Navarro \& Lorenzo, 2006). Quando se compara o tratamento com Peróxido de Carbamida a 10\% e 16\%, nota-se que há a mesma mudança de cor e o efeito de clareamento permanece estável por pelo menos um ano, sem necessitar de um novo clareamento, embora se possa observar alguma reversão do efeito com o Peróxido de Carbamida 16\% (Meireles et al., 2009).

Ao avaliar dois sistemas de clareamento dental caseiros, em diferentes tempos de uso diário (Whitness Perfect - Peróxido de Carbamida a 16\% durante 8h; e White Class - Peróxido de Hidrogênio com cálcio a 7,5\% durante 1h), observou-se o efeito clareador, a sensibilidade e a satisfação do paciente, e notou-se que não houve diferença significativa quanto à satisfação do resultado estético, sensibilidade, nem quanto à eficácia. Mas, apesar de não haver diferença estatística, o Peróxido de Carbamida 16\% alcançou as maiores médias de clareamento, isso porque o Peróxido de Carbamida forma a uréia como um de seus subprodutos que, teoricamente, pode se decompor ainda em dióxido de carbono e amônia, facilitando o procedimento de clareamento, devido ao pH elevado da amônia (Silva, Nacano \& Gava Pizi, 2012).

Almeida et al. (2015) avaliaram, in vitro, a alteração de cor dos dentes, a difusão do Peróxido de Hidrogênio e a citotoxicidade de clareadores caseiros contendo Peróxido de Hidrogênio a 6\% e Peróxido de Carbamida a 10\%, aplicados em tempos variados no substrato dental, para verificar se um tempo de exposição reduzido pode minimizar efeitos tóxicos sem prejudicar a eficácia do clareamento, e se o Peróxido de Hidrogênio a 6\% é menos tóxico do que o Peróxido de Carbamida a $10 \%$. Observaram que a redução do tempo de exposição diminuiu a eficácia dos tratamentos com os dois produtos; todos os protocolos foram eficazes, mas o tratamento com Peróxido de Carbamida a 10\% por três horas ao dia foi o melhor protocolo. A maior difusão de Peróxido de Hidrogênio não significou maior eficácia. Os protocolos avaliados não foram citotóxicos para células semelhantes a odontoblastos.

Durante um ensaio clínico randomizado sobre a eficácia e a segurança de quatro géis de clareamento caseiro (Peróxido de Carbamida a 10\%, Peróxido de Carbamida a 15\%, Peróxido de Hidrogênio a 7,5\%, Peróxido de Hidrogênio a 9,5\%) aplicados uma hora ao dia, durante 14 dias, foi observado que não houve diferença na intensidade da sensibilidade, e nem do grau de clareamento dos dentes (Peña \& Ratón, 2014). Entretanto, outro estudo demonstrou que o clareamento caseiro com Peróxido de Carbamida a $10 \%$ possui um risco e intensidade significativamente menor de sensibilidade dentária, quando comparado a concentrações mais altas do mesmo peróxido, sem comprometer a mudança de cor (Geus, Wambier, Boing, Loguercio \& Reis, 
2018). Assim sendo, indica-se o uso de géis em menor concentração, conforme recomendado pela American Dental Association e pelo Scientific Committee sobre produtos de consumo da Comissão Europeia (Peña \& Ratón, 2014).

Ao comparar as duas técnicas disponíveis, nota-se que não há diferença significativa entre o clareamento caseiro e o de consultório, quanto ao risco e intensidade de sensibilidade dentária, e eficácia (Geus, Wambier, Kossatz, Loguercio \& Reis, 2016). Já para outros autores, o clareamento de consultório apresenta uma sensibilidade inicial maior do que o caseiro (Mondelli, Azevedo, Francisconi, Almeida \& Ishikiriama, 2012).

Segundo Rodrigues et al., (2018), após uma sessão de clareamento de consultório, não há diferença na efetividade clareadora e na sensibilidade dentária em se realizar uma segunda sessão de consultório ou associá-la com uma semana de clareamento caseiro. Porém, outro estudo, mostrou que uma única sessão preliminar de clareamento em consultório com Peróxido de Hidrogênio a 35\% por 45min reduziu (em 3,7 dias) o tempo médio necessário para obter uma cor satisfatória usando Peróxido de Carbamida a 10\% por uma hora ao dia em casa. No entanto, o protocolo combinado resultou em um aumento do risco e nível de sensibilidade dentária, sem afetar as alterações finais de cor e o nível de satisfação dos pacientes com o sorriso (Vaez et al., 2019). Assim sendo, quando indicada corretamente, a combinação das técnicas de clareamento caseiro e de consultório pode ser uma escolha aceitável de tratamento, capaz de aprimorar a estética do sorriso com uma técnica simples que preserva a boa estrutura dentária. Ademais, o paciente deve ser orientado para a preservação máxima da cor e manutenção dos resultados estéticos, e de que os retratamentos podem ser necessários, dependendo da higiene bucal e da frequência da ingestão de alimentos e bebidas coloridos (Monteiro et al., 2020).

Assim, os dados acumulados nas últimas duas décadas demonstram que, quando usado adequadamente, o clareamento dental à base de peróxido é seguro e eficaz; no entanto, efeitos adversos podem ocorrer com aplicação, abuso ou uso inadequado de produtos de clareamento (Mchantaf et al., 2017). Nos dias atuais, o clareamento dental sem prescrição odontológica, como, fitas, pastas, ou até mesmo pós que prometem promover um sorriso branco de maneira simples e barata, vem sendo conhecido e utilizado por algumas pessoas. De acordo com uma pesquisa feita com os residentes da cidade de

Riyadh, Reino da Arábia Saudita (KSA), sobre clareamento de consultório versus o sem prescrição odontológica, avaliando a consciência dos mesmos sobre as indicações e os efeitos colaterais daqueles sem prescrição, observou-se que a maioria dos participantes já havia realizado o clareamento doméstico, sem indicação do cirurgião-dentista. No entanto, eles não estavam cientes da segurança dos produtos vendidos sem receita odontológica. Logo, nota-se que é necessário direcionar mais projetos comunitários para educar e aumentar a conscientização sobre as indicações e os efeitos colaterais pós-operatórios dos produtos de clareamento dental caseiro sem receita (Ahmed et al., 2020).

\section{Conclusão}

Com base nesse estudo, pode-se concluir que as três técnicas de clareamento dental (clareamento caseiro, de consultório ou combinação de ambos) conseguem atingir a mesma eficácia, quanto à alteração de cor, embora possam atingir os resultados em diferentes tempos clínicos e possam gerar diferentes graus de degradação do esmalte.

Quanto à concentração dos géis clareadores, observa-se que quanto mais alta a concentração, maior tende a ser o risco e a intensidade de sensibilidade dentária, devido a uma maior desmineralização do esmalte. Porém, para auxiliar na redução da sensibilidade dentária, o cirurgião-dentista pode fornecer ou aplicar um agente dessensibilizante; interromper o tratamento por um ou dois dias; ou utilizar géis que possuam cálcio ou fluoreto em sua composição.

Quanto ao clareamento de consultório, recomenda-se utilizar o protocolo de aplicação única, para géis de $\mathrm{pH}$ neutro/alcalino, visto que parece não haver alteração na eficácia e nem na sensibilidade dentária quando se faz aplicações 
múltiplas. Já para géis de pH ácido, sugere-se optar por múltiplas aplicações em tempo reduzido, a fim de minimizar a penetração do gel na câmara pulpar, reduzindo a sensibilidade dentária.

A utilização da técnica associada, na qual se realiza uma sessão preliminar de clareamento de consultório, combinada a sessões posteriores de clareamento caseiro, pode resultar na mesma eficácia de se caso não houvesse a sessão preliminar, porém, com maior risco de desenvolver sensibilidade dentária. Assim sendo, essa técnica normalmente é utilizada quando o paciente deseja acelerar o tratamento ou obter um resultado imediato, visto que essa associação acelera o processo de clareamento.

Quanto ao clareamento caseiro, sugere-se a utilização de um gel de Peróxido de Carbamida na menor concentração, pois o mesmo é eficaz quando comparado aos de maiores concentrações, e apresenta um risco menor de desenvolvimento de sensibilidade dentária.

Além disso, sugere-se que mais estudos comparando todas as técnicas de clareamento dental disponíveis na atualidade sejam realizados, a fim de comparar também outros parâmetros além da eficácia e sensibilidade, como por exemplo, a estabilidade de cor, a influência do consumo de diferentes produtos pigmentados e ácidos, e a relação entre o pH dos géis utilizados e a sua penetração na estrutura dental.

\section{Conflito de interesses}

Nenhum dos autores deste manuscrito possuem conflito de interesse de qualquer natureza com a produção deste artigo.

\section{Suporte financeiro}

Nenhum dos autores deste manuscrito receberam auxílio financeiro de qualquer natureza para sua produção.

\section{Referências}

Ahmed, Y. T., Al-Fkein, A. F., Alrejaie, L. M., Al Barazi, J. G., Alhaffar, D. R., Alqarni, G. S., \& Alhajibrahim, D. A. (2020). Evaluation and knowledge in choice of at home dental bleaching versus inoffice bleaching in Riyadh, Saudi Arabia. JAMDSR, 8(1): 23-26.

Almeida, L. C. A. G., Soares, D. G., Azevedo, F. A., Gallinari, M. O., Costa, C. A. S., Santos, P. H., \& Briso, A. L. F. (2015). At-Home Bleaching: Color Alteration, Hydrogen Peroxide Diffusion and Cytotoxicity. Braz Dent J, 26(4): 378-383.

Almeida, F. S. O., Fachiano, R. B., Thebaldo, J. D., Ramos-Tonello, C. M., Aguiar, F. H. B., Lima, D. A. N. L., \& Vieira-Juinor, W. F. (2021). Controle da sensibilidade dentária associada ao clareamento dental: relato de caso. Arch Health Invest, 10(1):94-99.

Araujo, J. L. S., Reis, B. S., Gonçalves, N. M., \& Brum, S.C. (2015). Técnicas de clareamento dental - revisão de literatura. Revista Pró-UniverSUS, 06(3): 3537.

Balladares, L., Alegria-Acevedo, L. F., Montenegro-Arana, A., Arana-Gordillo, L. A., Pulido, C., Salazar-Gracez, M. T., Reis, A., \& Loguercio, A. D. (2019). Effects of $\mathrm{pH}$ and Application Technique of In-office Bleaching Gels on Hydrogen Peroxide Penetration into the Pulp Chamber. Oper Dent, $44(6)$ : 659-667.

Baratieri, L. N., Junior, S. M., Andrada, M. A. C., \& Vieira, L. C. C. (1993). Clareamento dental. $1^{\text {a }}$ edição. São Paulo: Santos e Quintessence; 176p.

Caballero, A. B., Navarro, L. F. \& Lorenzo, J. A. (2006). At-home vital bleaching: a comparison of hydrogen peroxide and carbamide peroxide treatments. Med Oral Patol Oral Cir Bucal, 11(1): 94-99.

Demir, N.; Karci, M.; \& Ozcan, M. (2020). Effects of 16\% Carbamide Peroxide Bleaching on the Surface Properties of Glazed Glassy Matrix Ceramics. Biomed Res. Int., 2020, p. 1-7.

Estrela, C. (2018). Metodologia Científica: Ciência, Ensino, Pesquisa. Editora Artes Médicas.

Fernandes, R. A., Strazzi-Sahyon, H. B., Suzuki, T. Y. U., Briso, A. L. F., \& Santos, P. H. (2020). Effect of dental bleaching on the microhardness and surface roughness of sealed composite resins. Restor Dent Endod, 45(1):1-8.

Geus, J. L., Wambier, L. M., Boing, T. F., Loguercio, A. D., \& Reis, A. (2018). At-home Bleaching With $10 \%$ vs More Concentrated Carbamide Peroxide Gels: A Systematic Review and Meta-analysis. Oper Dent, 43(4): 210-222. 
Geus, J. L., Wambier, L. M., Kossatz, S., Loguercio, A. D., \& Reis, A. (2016). At-home vs In-office Bleaching: A Systematic Review and Meta-analysis. Oper Dent, 41(4): 341-356.

Gonçalves, M. L. L., Tavares, A. C. S., Mota, A. C. C., Penna, L. A. P., Deana, A. M., \& Bussadori, S. K. (2017). In-Office Tooth Bleaching for Adolescents Using Hydrogen Peroxide-Based Gels: Clinical Trial. Brazilian Dental Journal, 28(6): 720-725.

Lan, W. C., Yusuf, A. S. H., Syam, S., Natsir, N., Qiu, Y., Ruslin, M., \& Saito, T. (2019). A clinical evaluation of tooth bleaching treatment using a dualbarrel in-office whitening system. J Case Rep Dent Med, 1(3): 53-56.

Martins, I.E.B., Onofre, S., F, N., Martins, L. M., Montenegro, A., Arana-Gordilo, L. A., Reis, A., Loguercio, A. D., \& Silva, L. M. (2018). Effectiveness of In-office Hydrogen Peroxide with Two Different Protocols: A Two-center Randomized Clinical Trial. Oper dent, 43(4): 353-361.

Matias, B. A., Wang, G., Matis, J. I., Cook, N. B., \& Eckert, G. J. (2015). White Diet: Is It Necessary During Tooth Whitening? Oper Dent, 40(3): 235-40.

Mchantaf, E., Mansour, H., Sabbagh, J., Feghali, M., \& Mcconnell, R. J. (2017). Frequently Asked Questions about Vital Tooth Whitening. Dent Update, 44: $56-63$.

Meireles, S. S., Santos, I. S., Bona, A. D., \& Demarco, F.F. (2009). A double-blind randomized controlled clinical trial of 10 percent versus 16 percent carbamide peroxide tooth-bleaching agents One-year follow-up. JADA, 140(9): 1109-1117.

Mondelli, R. F. L., Azevedo, J. F. D. G., Francisconi, A. C., Almeida, C. M., \& Ishikiriama, S. K. (2012). Comparative clinical study of the effectiveness of different dental bleaching methods - two year follow-up. J Appl Oral Sci, 20(4): 435-43.

Monteiro, D., Vieira, A., Magalhães, C., Silva, N., \& Albuquerque, R. (2020). Combination of the custom trays bleaching technique with the in-office bleaching and considerations for result maintenance. Rev. Gaúch. Odontol, 68: 1-6.

Moradas, E. M. (2017) ¿Qué material y técnica seleccionamos a la hora de realizar un blanqueamiento dental y por qué? Protocolo para evitar hipersensibilidad dental posterior. Av. Odontoestomato, 33(3): 103-112.

Niessen, L. C. (2007). Talking with Patients Tooth Whitening: Why, Who, Where: What, and How. Jornaul of esthetic and restorative, 13(1): 79-80.

Palma, F. A. M., Abreu, G. B. A., Silva, T. M. R., Souza, V. A. R., Barbosa, E. S., Freire, G. S., Neves, G. S., Souza, M. M., Martim, L. \& Nahsan, F. P. S. (2021). Análise da utilização de dessensibilizante no uso prévio ao clareamento dentário: revisão narrativa. REAS, 13(5): 1-8.

Peixoto, A. C., Vaez, S. C., Pereira, N. A. R., Santana, C. N. S., Soares, K. D. A., Romão, A. C. T. R., Ferreira, L. F., Martins-Filho, P. R. S., \& Faria-e-Silva, A. L. (2018). High-concentration carbamide peroxide can reduce the sensitivity caused by in-office tooth bleaching: a single-blinded randomized controlled trial. J Appl Oral Sci, 26: 1-10.

Peña, V. A. \& Ratón, M. L. (2014). Randomized Clinical Trial on the Efficacy and Safety of Four Professional At-home Tooth Whitening Gels. Oper dent, 29(2): 136-143.

Rahal, V., Gallinari, M. O., Perdigão, J., Cintra, L. T. A., Dos Santos, P. H., \& Briso, A. L. F. (2015). Quantitative Sensory Testing of the Effect of Desensitizing Treatment After Dental Bleaching. Acta odontologica latinoamericana, 28(3): 263-270.

Rezende, M., Coppla, F. M., Chemin, K., Chibinski, A. C., Loguercio, A. D., \& Reis, A. (2019). Tooth Sensitivity After Dental Bleaching With a Desensitizercontaining and a Desensitizer-free Bleaching Gel: A Systematic Review and Meta-analysis. Oper Dent, 44(2): 58-74.

Rezende, M., Loguercio, A. D., Reis, A. \& Kossatz, S. (2013). Clinical Effects of Exposure to Coffee During At-home Vital Bleaching. Oper Dent, 38(6): 229-36.

Ribeiro, M. E. S., Santos, H. S. B., Baia, J. C. P., Oliveira, R. P., Junior, M. H. S. S., \& Loretto, S. C. (2019). Influence of prolonged tooth bleaching on enamel mass variation. Int. J. Odontostomat, 13(3): 305-309.

Rodrigues, J. L., Rocha, P. S., Pardim, S. L. S., Machado, A. C. V., Silva, A. L. F., \& Seraidarian, P. I. (2018). Association Between In-Office And At-Home Tooth Bleaching: A Single Blind Randomized Clinical Trial. Braz Dent J, 29(2): 133-139.

Silva, F. M. M.; Nacano, L. G.; \& Gava Pizi, E. C. (2012). Avaliação Clínica de Dois Sistemas de Clareamento Dental. Rev Odontol Bras Central, 21(56): 473-479.

Vaez, S. C., Correia, A. C. C., Santana, T. R., Santana, M. L. C., Peixoto, A. C., Leal, P. C., \& Faria-e-Silva, A. L. (2019). Is a Single Preliminary Session of Inoffice Bleaching Beneficial for the Effectiveness of At-home Tooth Bleaching? A Randomized Controlled Clinical Trial. Oper Dent, 44(4): 180-189.

Vieira, I., Vieira-Junior, W. F., Pauli, M. C., Theobaldo, J. D., Aguiar, F. H. B., Lima, D. A. N. L., \& Leonardi, G. R. (2020). Effect of in-office bleaching gels with calcium or fluoride on color, roughness, and enamel microhardness. J Clin Exp Dent, 12(2): 116-122. 